\title{
Correction to: Molecular identification of aflatoxigenic Aspergillus species in dried nuts and grains collected from Tehran, Iran
}

\author{
Arash Rahimi ${ }^{1}$ - Elahe Sasani ${ }^{2}$. Sassan Rezaie ${ }^{3} \cdot$ Mohammad Mehdi Soltan Dallal $^{1}$. Shahram Mahmoudi ${ }^{4}(\mathbb{D}$. \\ Ali Ahmadi ${ }^{3}$ - $\cdot$ Mansoureh Ghaffari ${ }^{5} \cdot$ Farzad Aala $^{6} \cdot$ Sadegh Khodavaisy $^{3}$ (i)
}

Published online: 19 October 2021

(c) Springer Nature Switzerland AG 2021

\section{Correction to: Journal of Environmental Health Science and Engineering https://doi.org/10.1007/s40201-021-00734-6}

The original article has been updated.

Publisher's note Springer Nature remains neutral with regard to jurisdictional claims in published maps and institutional affiliations.

The original article can be found online at https://doi.org/10.1007/ s40201-021-00734-6

Sadegh Khodavaisy

Sadegh_7392008@yahoo.com

1 Department of Pathobiology, School of Public Health, Tehran University of Medical Sciences, Tehran, Iran

2 Department of Medical Mycology, Faculty of Medical Sciences, Tarbiat Modares University, Tehran, Iran

3 Department of Medical Parasitology and Mycology, School of Public Health, Tehran University of Medical Sciences, Tehran, Iran

4 Department of Parasitology and Mycology, School of Medicine, Iran University of Medical Sciences, Tehran, Iran

5 Department of Microbiology, Faculty of Science, Islamic Azad University, Varamin-Pishva, Iran

6 Department of Parasitology and Mycology, Faculty of Medicine, Kurdistan University of Medical Sciences, Sanandaj, Iran 